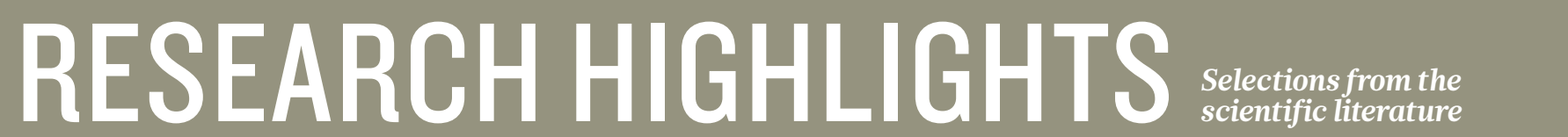

\section{HUMAN EVOLUTIOI}

\section{Interbreeding in ancient Africa}

Fossils from Africa exhibit features of both modern humans and archaic species - possible evidence for interbreeding. However, finding a genetic legacy of such encounters has been difficult because of a lack of ancient human DNA from Africa.

Michael Hammer at the University of Arizona in Tucson and his team analysed the entire genomes of seven individuals from two contemporary Western African Pygmy groups. The researchers identified 265 regions of the Pygmy genomes that may have been acquired through ancient interbreeding with other species, events that could have happened as recently as 9,000 years ago. Genome Res. http://doi.org/bct3 (2016)

\section{DEVELOPMENTAL BIOLOGY}

\section{Gene lets animals tell left from right}

A gene defines left and right during embryonic development in snails and frogs.

Animals generally look symmetrical, but internal organs are often positioned asymmetrically. To find out how embryos first define left and right at the molecular level, Angus Davison at the University of Nottingham, UK,

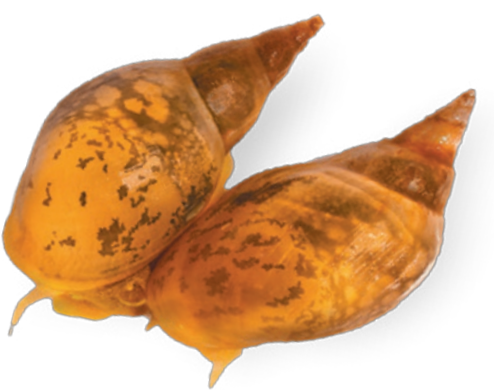

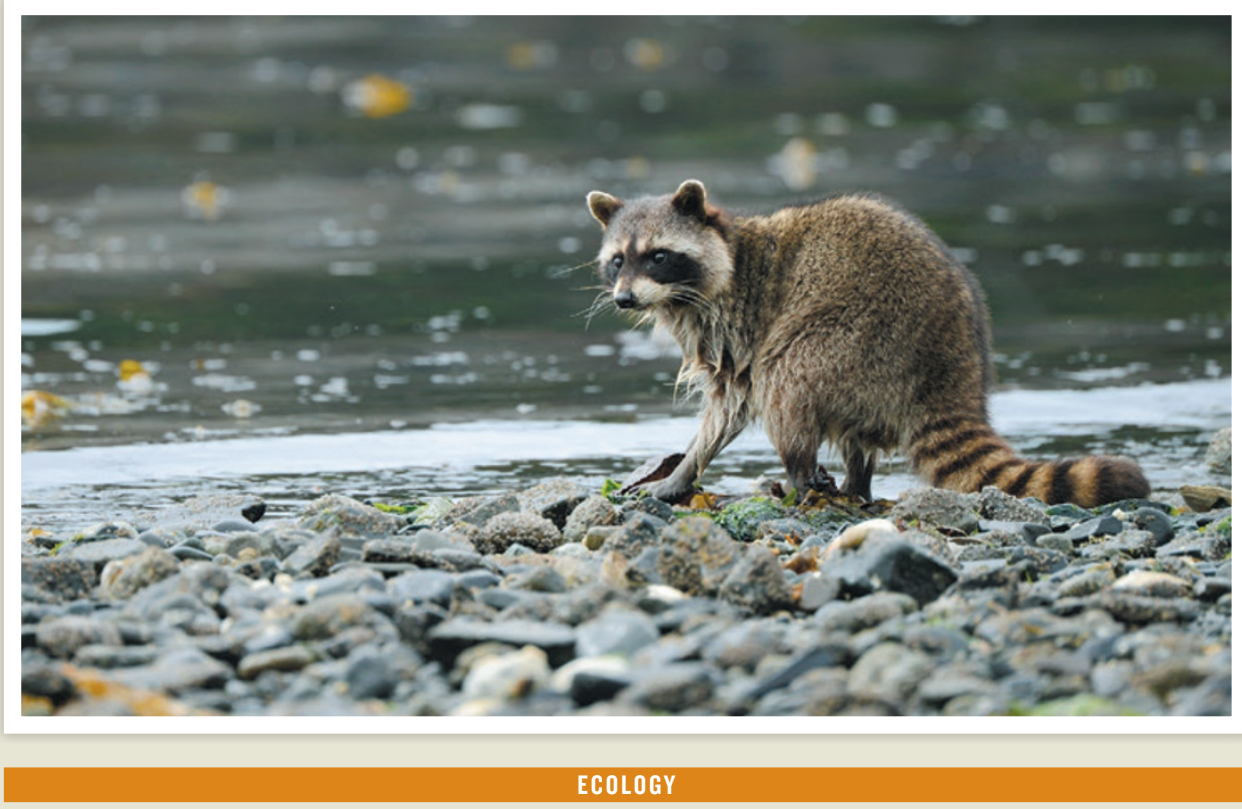

\section{Hearing is fearing for raccoons}

Fear of predators can trigger a cascade of effects through an ecosystem.

Humans have eliminated most predators of raccoons (Procyon lotor; pictured) - such as cougars and wolves - from the Gulf Islands of British Columbia in Canada. This has allowed the raccoons to forage almost freely on shoreline species such as crabs and fish. To instil fear in the raccoons, Justin Suraci at the University of Victoria in Canada and his colleagues broadcast dog vocalizations over various island shores for one month. They found that foraging by racoons decreased drastically at these locations compared to areas where seal vocalizations were broadcast. This caused the number of some crabs to increase by up to $97 \%$ and numbers of some fish to rise by $81 \%$. The snail prey of one crab species saw declines.

This manipulation of fear shows the cascading effects of losing large predators from ecosystems, the authors say.

Nature Commun. 7, 10698 (2016) and his colleagues compared the DNA of pond snails (Lymnaea stagnalis; pictured) that had shells with clockwise or anti-clockwise spirals.

They found that formin, a cell-structure protein, was consistently linked to spiral direction and is expressed early in snail development, showing asymmetry even in two-cell embryos.

The team treated frog embryos (Xenopus laevis) with anti-formin drugs, and found that $13 \%$ developed an organ on the opposite side to its normal position, suggesting that formin also coordinates this process in frogs. Curr. Biol. http://dx.doi.org/ 10.1016/j.cub.2015.12.071(2016)

\section{CLIMATE SCIENCE \\ Where climate models fall short}

Climate models tend to overestimate the extent to which climate change contributes to weather events such as extreme heat and rain.

Omar Bellprat and Francisco Doblas-Reyes at the Catalan Institute of Climate Sciences in Barcelona, Spain, used an idealized statistical model to compare the frequency of weather extremes in simulations with and without climate warming. Extreme events seemed to be more closely linked to climate change when the model was forced to run at low levels of reliability than when the model error was kept to a minimum.

To account for models' biased representation of climate variability, studies should rely on calibrated model ensembles, which are commonly used by weather forecasters, the authors suggest.

Geophys. Res. Lett. http://doi. org/bcsr (2016) 